General Mathematics Vol. 27, No. 2 (2019), 85-95

DOI:10.2478/gm-2019-0017

S sciendo

\title{
Quantitative results for positive linear operators which preserve certain functions ${ }^{1}$
}

\author{
Marius-Mihai Birou
}

\begin{abstract}
In this paper we obtain estimations of the errors in approximation by positive linear operators which fix certain functions. We use both the first and the second order classical moduli of smoothness and a generalized modulus of continuity of order two. Some applications involving Bernstein type operators, Kantorovich type operators and genuine Bernstein-Durrmeyer type operators are presented.
\end{abstract}

2010 Mathematics Subject Classification: 41A36, 41A25.

Key words and phrases: positive linear operators, errors estimations, classical moduli of smoothness, generalized modulus of continuity, Kantorovich type operators, genuine Bernstein-Durrmeyer type operators.

\footnotetext{
${ }^{1}$ Received 24 October, 2019

Accepted for publication (in revised form) 18 November, 2019
} 


\section{Introduction}

Let $n \in \mathbb{N}, n \geq 1$. The classical Bernstein operator is given by

$$
B_{n}(f)(x)=\sum_{k=0}^{n} p_{n, k}(x) f\left(\frac{k}{n}\right), x \in[0,1], f \in C[0,1],
$$

where

$$
p_{n, k}(x)=\left(\begin{array}{l}
n \\
k
\end{array}\right) x^{k}(1-x)^{n-k}, k=0, \ldots, n .
$$

It preserves the functions $e_{i}, i=0,1$ where $e_{i}(x)=x^{i}, i=0,1, \ldots$ (i.e. the operator fixes linear functions).

Let $\tau:[0,1] \rightarrow[0,1]$ a function with the properties:

i) $\tau$ is $\infty$-times continuously differentiable;

ii) $\tau(0)=0, \tau(1)=1$ and $\tau^{\prime}(x)>0, x \in[0,1]$.

In [1] the authors considered the Bernstein type operators

$B_{n}^{\tau}(f)(x)=\sum_{k=0}^{n}\left(\begin{array}{l}n \\ k\end{array}\right) \tau(x)^{k}(1-\tau(x))^{n-k}\left(f \circ \tau^{-1}\right)\left(\frac{k}{n}\right), x \in[0,1], f \in C[0,1]$

or equivalent

$$
B_{n}^{\tau}(f)=B_{n}\left(f \circ \tau^{-1}\right) \circ \tau .
$$

The operators $B_{n}^{\tau}$ have the properties

$$
B_{n}^{\tau}\left(e_{0}\right)=e_{0}, B_{n}^{\tau}(\tau)=\tau, B_{n}^{\tau}(\tau)=\tau^{2}+\frac{\tau(1-\tau)}{n} .
$$

The set $\left\{1, \tau, \tau^{2}\right\}$ is a Extended Complete Chebyshev system (for more about Chebyshev systems the reader can consult [5]). We get, for every $f \in$ $C[0,1]$,

$$
\lim _{n \rightarrow \infty} B_{n}^{\tau}(f)=f, \text { uniformly on }[0,1] .
$$

The following estimation of the approximation error was given in [1]. 
Theorem 1 For every $f \in C[0,1]$ and $\delta>0$, we have

$$
\left|f(x)-B_{n}^{\tau}(f)(x)\right| \leq\left(1+\frac{\tau^{\prime}(x) \tau(x)(1-\tau(x))}{n K_{\tau} \delta^{2}}\right) \omega_{1}(f, \delta), x \in[0,1],
$$

where $\omega_{1}$ is the classical modulus of continuity of order one, i.e.

$$
\omega_{1}(f, \delta)=\sup \{f(x+h)-f(x): x, x+h \in[0,1], 0 \leq h \leq \delta\} .
$$

The constant $K_{\tau}$ from estimation (2) satisfy the condition

$$
(x-t)^{2} K_{\tau} \leq \tau^{\prime}(x)(\tau(t)-\tau(x))^{2}, x, t \in[0,1] .
$$

The existence of the constant $K_{\tau}$ from (3) is proved by Freud in [3]. For $\tau=e_{1}$ we have $K_{\tau}=1$.

Throughout the paper $\tau:[0,1] \rightarrow[0,1]$ is a function having the properties i) and ii) and $K_{\tau}$ is a constant which satisfies the condition (3).

In Section 2 we give errors estimations in approximation by positive linear operators which preserve the functions $e_{0}$ and $\tau$. We use the classical moduli of smoothness of order one and two. We also get a quantitative result using a generalized modulus of continuity of order two introduced in [7]. The Section 3 contains applications for the operators $B_{n}^{\tau}$, some Kantorovich type operators and some genuine Bernstein-Durrmeyer operators. Also, a class of Kantorovich type operators which preserve linear functions is presented.

\section{Quantitative results}

For every $f \in C[0,1]$ and $\delta>0$ the second order modulus of continuity is defined by

$$
\omega_{2}(f, \delta)=\sup \{f(x+h)-2 f(x)+f(x-h): x, x \pm h \in[0,1], 0 \leq h \leq \delta\} .
$$

Theorem 2 Let $L^{\tau}: C[0,1] \rightarrow C[0,1]$ a positive linear operator which preserves $e_{0}$ and $\tau$. Then, for every $f \in C[0,1]$ and $\delta>0$, we have the estimation

$$
\left|f(x)-L^{\tau}(f)(x)\right| \leq\left(1+\frac{G_{\tau}(x)}{\delta^{2}}\right) \omega_{1}(f, \delta), x \in[0,1],
$$

where

$$
G_{\tau}(x)=\frac{1}{K_{\tau}}\left(L^{\tau}\left(\tau^{2}\right)(x)-\tau^{2}(x)\right) \tau^{\prime}(x) .
$$


Proof. Let $f \in C[0,1], x \in[0,1]$ and $\delta>0$. From [8] we have

$$
\begin{aligned}
& \left|f(x)-L^{\tau}(f)(x)\right| \leq|f(x)|\left|L^{\tau}\left(e_{0}\right)(x)-e_{0}(x)\right| \\
& +\left(L^{\tau}\left(e_{0}\right)(x)+\frac{L^{\tau}\left(e_{1}-x e_{0}\right)^{2}(x)}{\delta^{2}}\right) \omega_{1}(f, \delta) .
\end{aligned}
$$

Using (3) and the preservation properties of the operator $L_{n}^{\tau}$ we obtain

$$
L^{\tau}\left(e_{1}-x e_{0}\right)^{2}(x) \leq \frac{\tau^{\prime}(x)}{K_{\tau}} L^{\tau}\left(\tau-\tau(x) e_{0}\right)^{2}(x)=G_{\tau}(x) .
$$

The conclusion follows from (5), (6) and taking into account that the operator $L^{\tau}$ fixes the constant functions.

Remark 1 If we take $L^{\tau}=B_{n}^{\tau}, n \geq 1$, then we get estimation (2).

Theorem 3 Let $L^{\tau}: C[0,1] \rightarrow C[0,1]$ a positive linear operators which preserves $e_{0}$ and $\tau$. Then, for every $f \in C[0,1]$ and $0<\delta \leq 1 / 2$, we get

$$
\left|f(x)-L^{\tau}(f)(x)\right| \leq \frac{\sqrt{G_{\tau}(x)}}{\delta} \omega_{1}(f, \delta)+\left(1+\frac{G_{\tau}(x)}{2 \delta^{2}}\right) \omega_{2}(f, \delta), x \in[0,1],
$$

where $G_{\tau}$ is given by (4).

Proof. Let $f \in C[0,1], x \in[0,1]$ and $0<\delta \leq 1 / 2$. From [6] we have

$$
\begin{gathered}
\left|f(x)-L^{\tau}(f)(x)\right| \leq|f(x)|\left|L^{\tau}\left(e_{0}\right)(x)-e_{0}(x)\right|+\frac{\left|L^{\tau}\left(e_{1}-x e_{0}\right)(x)\right|}{\delta} \omega_{1}(f, \delta) \\
+\left(L^{\tau}\left(e_{0}\right)(x)+\frac{L^{\tau}\left(e_{1}-x e_{0}\right)^{2}(x)}{2 \delta^{2}}\right) \omega_{2}(f, \delta) .
\end{gathered}
$$

Using Cauchy-Schwarz inequality and (6) we get

(8) $\left|L^{\tau}\left(e_{1}-x e_{0}\right)(x)\right| \leq L^{\tau}\left(\left|e_{1}-x e_{0}\right|\right)(x) \leq \sqrt{L^{\tau}\left(e_{1}-x e_{0}\right)^{2}(x)} \leq \sqrt{G_{\tau}(x)}$.

Using (7), (8) and the fact that the operator $L^{\tau}$ preserves the constant functions we get the conclusion.

Next we present some notations, definitions and results from [7].

Let $x \in(0,1)$ and $\nu, \eta, \sigma \in C[0,1]$ having the properties

i) $\min _{t \in[0,1]} \nu(t)=\nu(x)=1$; 
ii) $\eta(t)<0, t \in[0, x)$ and $\eta(x)=0$ and $\eta(t)>0, t \in(x, 1]$;

iii) $\sigma(t)>0, t \in[0,1] \backslash\{x\}$ and $\sigma(x)=0$;

iv) $\{\nu, \eta, \sigma\}$ is an Extended Complete Chebyshev system on $[0,1]$.

We have the notations

$$
\begin{gathered}
l(t)=\frac{\eta(t)}{\nu(t)}, t \in[0,1], \\
d(u, v)=|l(v)-l(u)|, u, v \in[0,1], \\
D(u, v)=\left|\begin{array}{cc}
\nu(u) & \nu(v) \\
\eta(u) & \eta(v)
\end{array}\right|, u, v \in[0,1] .
\end{gathered}
$$

Definition 1 [Y] If $f:[0,1] \rightarrow \mathbb{R}$ and $\delta>0$ we define

$$
\omega_{1}^{*}(f, \delta)=\sup \left\{\left|\frac{f(u)}{\nu(u)}-\frac{f(v)}{\nu(v)}\right|, u, v \in[0,1], d(u, v) \leq \delta\right\}
$$

and

(9)

$$
\omega_{2}^{*}(f, \delta)=\sup \{|\Delta(f ; u, v, w)|, 0 \leq u<v<w \leq 1, d(u, v) \leq \delta, d(v, w) \leq \delta\},
$$

where

$$
\Delta(f ; u, v, w)=\frac{D(v, w)}{D(u, w)} f(u)+\frac{D(u, v)}{D(u, w)} f(w)-f(v)
$$

Definition 2 [7] For $0<\delta \leq d(0,1) / 2$ we define

$$
\Psi(\delta)=\inf \{|\Delta(\sigma ; u, v, w)|, 0 \leq u<v<w \leq 1, d(u, v)=d(v, w)=\delta\}
$$

Theorem 4 [7] Let $L: C[0,1] \rightarrow C[0,1]$ a positive linear operator. Then, for any $f \in C[0,1], x \in[0,1]$ and $0<\delta \leq d(0,1) / 2$, we have

$$
\begin{gathered}
|L(f)(x)-f(x)| \leq|f(x)||L(\nu)(x)-1|+\frac{1}{\delta}|L(\eta)(x)| \omega_{1}^{*}(f, \delta) \\
+\left(2 L(\nu)(x)+\frac{L(\sigma)(x)}{\Psi(\delta)}\right) \omega_{2}^{*}(f, \delta)
\end{gathered}
$$


Theorem 5 Let $L^{\tau}: C[0,1] \rightarrow C[0,1]$ a positive linear operator which preserves $e_{0}$ and $\tau$. Then, for any $f \in C[0,1], x \in[0,1]$ and $0<\delta \leq 1 / 2$, we have

$$
\left|L^{\tau}(f)(x)-f(x)\right| \leq\left(2+\frac{L^{\tau}\left(\tau^{2}\right)(x)-\tau^{2}(x)}{\delta^{2}}\right) \omega_{2, \tau}^{*}(f, \delta),
$$

where

(11)

$$
\begin{gathered}
\omega_{2, \tau}^{*}(f, \delta)= \\
\sup \{|\Delta(f ; u, v, w)|, 0 \leq u<v<w \leq 1, \tau(v)-\tau(u) \leq \delta, \tau(w)-\tau(v) \leq \delta\}
\end{gathered}
$$

with

$$
\Delta(f ; u, v, w)=\frac{\tau(w)-\tau(v)}{\tau(w)-\tau(u)} f(u)+\frac{\tau(v)-\tau(u)}{\tau(w)-\tau(u)} f(w)-f(v) .
$$

Proof. Let $f \in C[0,1]$ and $0<\delta \leq 1 / 2$.

If $x \in\{0,1\}$, then $L^{\tau}(f)(x)=f(x)$. Thus the estimation (10) holds.

Let $x \in(0,1)$. We use Theorem 4 with

$$
\nu=e_{0}, \eta=\tau-\tau(x) e_{0}, \sigma=\left(\tau-\tau(x) e_{0}\right)^{2} .
$$

It is easy to check that the functions given by (12) satisfy the properties i)-iv) from this section.

We also have

$$
\begin{gathered}
l(t)=\tau(t)-\tau(x), t \in[0,1], \\
d(s, t)=\tau(t)-\tau(s), 0 \leq s<t \leq 1, \\
D(s, t)=\tau(t)-\tau(s), s, t \in[0,1] .
\end{gathered}
$$

It follows that $\omega_{2}^{*}(f, \delta)$ given by $(9)$ becomes $\omega_{2, \tau}^{*}(f, \delta)$ from $(11)$.

As $\Psi(\delta)=\delta^{2}$ and using the preservation properties of the operator $L^{\tau}$ we get the conclusion.

\section{Applications}

\subsection{The operators $B_{n}^{\tau}$}

Let $B_{n}^{\tau}$ the operator given by (1). 
Theorem 6 For every $f \in C[0,1]$ we have

$$
\begin{array}{r}
\left|f(x)-B_{n}^{\tau}(f)(x)\right| \leq \sqrt{\frac{\tau^{\prime}(x)}{K_{\tau}}} \omega_{1}\left(f, \sqrt{\frac{\tau(x)(1-\tau(x))}{n}}\right) \\
+\left(1+\frac{\tau^{\prime}(x)}{2 K_{\tau}}\right) \omega_{2}\left(f, \sqrt{\frac{\tau(x)(1-\tau(x))}{n}}\right), x \in[0,1]
\end{array}
$$

and

$$
\left|f(x)-B_{n}^{\tau}(f)(x)\right| \leq 3 \omega_{2, \tau}^{*}\left(f, \sqrt{\frac{\tau(x)(1-\tau(x))}{n}}\right), x \in[0,1] .
$$

Proof. We use Theorem 3 and Theorem 5 with

$$
\delta=\sqrt{\frac{\tau(x)(1-\tau(x))}{n}} .
$$

\subsection{Kantorovich type operators}

Let $a_{n} \in \mathbb{R}^{+}, n \geq 1$ with $a_{n} \leq 1 / n$. Let $f:[0,1] \rightarrow \mathbb{R}$ be a continuous function in $x=0,1$ and $f \in L_{1}\left[-a_{n}+k / n, a_{n}+k / n\right], k=1,2, \ldots, n-1$.

For $x \in[0,1]$, we define the positive linear operators

$$
K_{n}(f)(x)=p_{n, 0}(x) f(0)+p_{n, n}(x) f(1)+\frac{1}{2 a_{n}} \sum_{k=1}^{n-1} p_{n, k}(x) \int_{-a_{n}+k / n}^{a_{n}+k / n} f(t) d t .
$$

It is easy to check that

$$
K_{n}\left(e_{0}\right)(x)=1, K_{n}\left(e_{1}\right)(x)=x, K_{n}\left(e_{2}\right)(x)=x^{2}+\frac{x(1-x)}{n}+\frac{a_{n}^{2}}{3}\left(1-x^{n}-(1-x)^{n}\right) .
$$

We observe that the operators $K_{n}$ reproduce the linear functions.

If $f \in C[0,1]$, then it follows

$$
\lim _{n \rightarrow \infty} K_{n}(f)=f, \text { uniformly on }[0,1] .
$$

Theorem 7 Let $f \in C[0,1]$ and

$$
\delta_{n}^{K}(x)=\sqrt{\frac{x(1-x)}{n}+\frac{a_{n}^{2}}{3}\left(1-x^{n}-(1-x)^{n}\right)}, x \in[0,1] .
$$


We have the estimations

$$
\left|f(x)-K_{n}(f)(x)\right| \leq 2 \omega_{1}\left(f, \delta_{n}^{K}(x)\right), x \in[0,1]
$$

and

$$
\left|f(x)-K_{n}(f)(x)\right| \leq \frac{3}{2} \omega_{2}\left(f, \delta_{n}^{K}(x)\right), x \in[0,1] .
$$

Proof. The estimations (14) and (15) follow using (13) and the estimations (5) and (7) respectively.

For every $f \in C[0,1]$ we define

$$
K_{n}^{\tau}(f)=K_{n}\left(f \circ \tau^{-1}\right) \circ \tau .
$$

i.e.

$$
\begin{gathered}
K_{n}^{\tau}(f)(x)=p_{n, 0}(\tau(x)) f(0)+p_{n, n}(\tau(x)) f(1) \\
+\frac{1}{2 a_{n}} \sum_{k=1}^{n-1} p_{n, k}(\tau(x)) \int_{-a_{n}+k / n}^{a_{n}+k / n} f\left(\tau^{-1}(t)\right) d t, x \in[0,1] .
\end{gathered}
$$

We have

$K_{n}^{\tau}\left(e_{0}\right)=e_{0}, K_{n}^{\tau}(\tau)=\tau, K_{n}^{\tau}\left(\tau^{2}\right)=\tau^{2}+\frac{\tau(1-\tau)}{n}+\frac{a_{n}^{2}}{3}\left(1-\tau^{n}-(1-\tau)^{n}\right)$.

If $f \in C[0,1]$, then it follows

$$
\lim _{n \rightarrow \infty} K_{n}^{\tau}(f)=f, \quad \text { uniformly on }[0,1] .
$$

\section{Theorem 8 Let}

$$
\delta_{n, \tau}^{K}(x)=\sqrt{\frac{\tau(x)(1-\tau(x))}{n}+\frac{a_{n}^{2}}{3}\left(1-\tau(x)^{n}-(1-\tau(x))^{n}\right)}, x \in[0,1]
$$

For every $f \in C[0,1]$ we get the estimations

$$
\left|f(x)-K_{n}^{\tau}(f)(x)\right| \leq\left(1+\frac{\tau^{\prime}(x)}{K_{\tau}}\right) \omega_{1}\left(f, \delta_{n, \tau}^{K}(x)\right), x \in[0,1],
$$

$\left|f(x)-K_{n}^{\tau}(f)(x)\right| \leq \sqrt{\frac{\tau^{\prime}(x)}{K_{\tau}}} \omega_{1}\left(f, \delta_{n, \tau}^{K}(x)\right)+\left(1+\frac{\tau^{\prime}(x)}{2 K_{\tau}}\right) \omega_{2}\left(f, \delta_{n, \tau}^{K}(x)\right), x \in[0,1]$ and

$$
\left|f(x)-K_{n}^{\tau}(f)(x)\right| \leq 3 \omega_{2}^{*}\left(f, \delta_{n, \tau}^{K}(x)\right), x \in[0,1] .
$$

Proof. The estimations (16), (17) and (18) follow using Theorem 2, Theorem 3 and Theorem 5 respectively with $\delta=\delta_{n, \tau}^{K}(x)$. 


\subsection{Genuine Bernstein-Durrmeyer type operators}

The genuine-Bernstein-Durrmeyer operator was introduced in [2] and [4].

For $f \in C[0,1]$ and $x \in[0,1]$ it is defined by

$$
U_{n}(f)(x)=p_{n, 0}(x) f(0)+p_{n, n}(x) f(1)+(n-1) \sum_{k=1}^{n-1} p_{n, k}(x) \int_{0}^{1} p_{n-2, k-1}(x) f(t) d t .
$$

We have

$$
U_{n}\left(e_{0}\right)(x)=1, U_{n}\left(e_{1}\right)(x)=x, U_{n}\left(e_{2}\right)(x)=x^{2}+\frac{2 x(1-x)}{n+1} .
$$

If $f \in C[0,1]$, then we get

$$
\lim _{n \rightarrow \infty} U_{n}(f)=f, \quad \text { uniformly on }[0,1] .
$$

For every $f \in C[0,1]$ we define

$$
U_{n}^{\tau}(f)=U_{n}\left(f \circ \tau^{-1}\right) \circ \tau
$$

i.e.

$$
\begin{gathered}
U_{n}^{\tau}(f)(x)=p_{n, 0}(\tau(x)) f(0)+p_{n, n}(\tau(x)) f(1) \\
+(n-1) \sum_{k=1}^{n-1} p_{n, k}(\tau(x)) \int_{0}^{1} p_{n-2, k-1}(x) f\left(\tau^{-1}(t)\right) d t, x \in[0,1] .
\end{gathered}
$$

We have

$$
U_{n}^{\tau}\left(e_{0}\right)=e_{0}, U_{n}^{\tau}(\tau)=\tau, U_{n}^{\tau}(\tau)=\tau^{2}+\frac{2 \tau(1-\tau)}{n+1} .
$$

If $f \in C[0,1]$, then it follows

$$
\lim _{n \rightarrow \infty} U_{n}^{\tau}(f)=f, \quad \text { uniformly on }[0,1] .
$$

Theorem 9 For every $f \in C[0,1]$ we get the estimations

$$
\begin{gathered}
\left|f(x)-U_{n}^{\tau}(f)(x)\right| \leq\left(1+\frac{\tau^{\prime}(x)}{K_{\tau}}\right) \omega_{1}\left(f, \sqrt{\frac{2 \tau(x)(1-\tau(x))}{n+1}}\right), x \in[0,1], \\
\left|f(x)-U_{n}^{\tau}(f)(x)\right| \leq \sqrt{\frac{\tau^{\prime}(x)}{K_{\tau}}} \omega_{1}\left(f, \sqrt{\frac{2 \tau(x)(1-\tau(x))}{n+1}}\right)
\end{gathered}
$$




$$
+\left(1+\frac{\tau^{\prime}(x)}{2 K_{\tau}}\right) \omega_{2}\left(f, \sqrt{\frac{2 \tau(x)(1-\tau(x))}{n+1}}\right), x \in[0,1]
$$

and

$$
\left|f(x)-U_{n}^{\tau}(f)(x)\right| \leq 3 \omega_{2}^{*}\left(f, \sqrt{\frac{2 \tau(x)(1-\tau(x))}{n+1}}\right), x \in[0,1] .
$$

Proof. We use the estimations from Theorem 2, Theorem 3 and Theorem 5 with

$$
\delta=\sqrt{\frac{2 \tau(x)(1-\tau(x))}{n+1}} .
$$

\section{References}

[1] D. Cardenas-Morales, P. Garrancho, I. Raşa, Bernstein-type operators which preserve polynomials, Comput. Math. Appl. 62, 2011, 158-163.

[2] W. Chen: On the modified Bernstein-Durrmeyer operators, Report of the Fifth Chinese Conference on Approximation Theory, Zhen Zhou, China, 1987.

[3] G. Freud, On approximation by positive linear methods I, II, Stud. Sci. Math. Hung. 2, 1967, 63-66; 3, 1968, 365-370.

[4] T.N.T. Goodman, A. Sharma, A modified Bernstein-Schoenberg operator, in: Proc. of the Conference on Constructive Theory of Functions, Varna 1987 (ed. by Sendov et al.), Sofia Publ. House Bulg. Acad. of Sci. 1988, 166-173.

[5] S. Karlin, W. Studden, Tchebycheff Systems: with Applications in Analysis and Statistics, Interscience, New York, 1966.

[6] R. Paltanea, Optimal estimates with modulus of continuity, Result. Math. 32, 1997, 318-331.

[7] R. Paltanea, Estimates with adapted moduli of continuity for a Chebyshev system, Proc. of Int. Conf. Numerical Analysis and Approximation Theory (O. Agratini and P. Blaga Eds), Cluj-Napoca, July 4-8, 2006, $337-352$. 
[8] O. Shisha, B. Mond, The degree of convergence of sequences of linear positive operators, Proc. Nat. Acad. Sci. U.S.A. 60, 1968, 1196-1200.

\section{Marius-Mihai Birou}

Technical University of Cluj Napoca, România

Faculty of Automatics and Computer Science

Department of Mathematics

28 Memorandumului Str., Cluj Napoca, România

e-mail: Marius.Birou@math.utcluj.ro 